KUCP-0138

hep-th/9906210

\title{
Baryon Configurations in the UV and IR Regions of Type 0 String Theory
}

\author{
Shigenori Seki ${ }^{\dagger}$ \\ Graduate School of Human and Environmental Studies \\ Kyoto University, Kyoto 606-8501, Japan
}

\begin{abstract}
The Type 0 string theory is considered as a dual model of a non-supersymmetric gauge theory. A background geometry with $N$ electric D3-branes is calculated in UV/IR regions. In this paper, we study a D5-brane around $N$ D3-branes from the D5-brane world volume action as in the Type IIB case, and we obtain some baryon configurations at UV/IR regions.
\end{abstract}

$6 / 99$

$\dagger$ seki@phys.h.kyoto-u.ac.jp 


\section{Introduction}

From the AdS/CFT correspondence, the Type IIB string theory with large N D3branes is believed to be a dual model of the $\mathcal{N}=4$ supersymmetric gauge theory, and a baryon configuration is realized as a D5-brane wrapped on $S^{5}$ in the Type IIB theory [1] [2]. In [3], the D5-brane world volume action is studied and the static solution is analysed. It has a spike, where the tension (the energy per unit radial coordinate distance) is equal to that of $N$ fundamental strings [4]. It is a bound state of $N$ quarks, that is a baryon.

Recently a Type 0 string theory has been considered as a dual model of a nonsupersymmetric gauge theory [5]. The Type 0 string theory has two NS-NS and two $\mathrm{R}-\mathrm{R}$ sectors and is classified into two types of models [6],

$$
\begin{array}{ll}
\text { Type 0A : } & \left(\mathrm{NS}-, \mathrm{NS}_{-}\right) \oplus(\mathrm{NS}+, \mathrm{NS}+) \oplus(\mathrm{R}+, \mathrm{R}-) \oplus(\mathrm{R}-, \mathrm{R}+), \\
\text { Type 0B : } & (\mathrm{NS}-, \mathrm{NS}-) \oplus(\mathrm{NS}+, \mathrm{NS}+) \oplus(\mathrm{R}+, \mathrm{R}+) \oplus(\mathrm{R}-, \mathrm{R}-) .
\end{array}
$$

The signs $( \pm)$ represent chiralities and the (NS-,NS-) sector has tachyon " $T$ ". But after tachyon condensation, tachyon mass squared is shifted by the five-form field strength. Since there are two R-R sectors, RR fields are two sets, electric and magnetic.

In the Type 0B theory, the system with large $N$ electric D3-branes is studied in [7] [8] [9]. The metric is set in a form

$$
d s^{2}=e^{\frac{1}{2} \phi}\left(e^{\frac{1}{2} \xi-5 \eta} d \rho^{2}+e^{-\frac{1}{2} \xi}\left(-d t^{2}+\sum_{i=1}^{3} d x_{i} d x_{i}\right)+e^{\frac{1}{2} \xi-\eta} d \Omega_{5}^{2}\right) .
$$

The D3-branes coordinates are $\left(t, x_{1}, x_{2}, x_{3}\right)$. Then the action is represented as

$$
\begin{aligned}
& S=\int d \rho\left(\frac{1}{2} \dot{\phi}^{2}+\frac{1}{2} \dot{\xi}^{2}+\frac{1}{4} \dot{T}^{2}-5 \dot{\eta}^{2}-V(\phi, \xi, \eta, T)\right), \\
& V(\phi, \xi, \eta, T)=\frac{1}{2} T^{2} e^{\frac{1}{2} \phi+\frac{1}{2} \xi-5 \eta}+20 e^{-4 \eta}-\frac{Q^{2}}{f(T)} e^{-2 \xi}, \\
& f(T)=1+T+\frac{1}{2} T^{2} .
\end{aligned}
$$

This is a toda like system.

The asymptotic solutions in the UV region $\left(\rho \equiv e^{-y} \ll 1\right)$ are analysed

$$
\begin{aligned}
T & =-1+\frac{8}{y}+\frac{4}{y^{2}}(39 \ln y-20)+O\left(\frac{\ln ^{2} y}{y^{3}}\right), \\
\phi & =\ln \left(2^{15} Q^{-1}\right)-2 \ln y+\frac{1}{y} 39 \ln y+O\left(\frac{\ln y}{y^{2}}\right), \\
\xi & =\ln (2 Q)-y+\frac{1}{y}+\frac{1}{2 y^{2}}(39 \ln y-104)+O\left(\frac{\ln ^{2} y}{y^{3}}\right), \\
\eta & =\ln 2-\frac{1}{2} y+\frac{1}{y}+\frac{1}{2 y^{2}}(39 \ln y-38)+O\left(\frac{\ln ^{2} y}{y^{3}}\right),
\end{aligned}
$$


where $Q$ is a D3-brane charge. At the UV limit $(\rho \rightarrow 0)$, the metric (1.1) becomes

$$
\begin{array}{r}
d s^{2}=R_{U V}^{2}\left(\frac{d v^{2}}{v^{2}}+\frac{v^{2}}{R_{U V}^{4}}\left(-d t^{2}+\sum_{i=1}^{3} d x_{i} d x_{i}\right)+d \Omega_{5}\right) \\
\phi=\phi_{0}, \quad R_{U V}^{2}=2^{-\frac{1}{2}} Q^{\frac{1}{2}} e^{\frac{1}{2} \phi_{0}}, \quad v=2^{-\frac{1}{2}} e^{\frac{1}{2} \phi_{0}} \rho^{-\frac{1}{4}}
\end{array}
$$

and this represents $A d S_{5} \times S^{5}$. On the other hand, the asymptotic solutions in the IR region $\left(\rho \equiv e^{y} \gg 1\right)$ are evaluated as

$$
\begin{aligned}
T & =-\frac{16}{y}-\frac{8}{y^{2}}(9 \ln y-3)+O\left(\frac{\ln ^{2} y}{y^{3}}\right) \\
\phi & =-\frac{1}{2} \ln \left(2 Q^{2}\right)+2 \ln y-\frac{1}{y} 9 \ln y+O\left(\frac{\ln y}{y^{2}}\right) \\
\xi & =\frac{1}{2} \ln \left(2 Q^{2}\right)+y+\frac{9}{y}+\frac{9}{2 y^{2}}\left(9 \ln y-\frac{20}{9}\right)+O\left(\frac{\ln ^{2} y}{y^{3}}\right) \\
\eta & =\ln 2+\frac{1}{2} y+\frac{1}{y}+\frac{1}{2 y^{2}}(9 \ln y-2)+O\left(\frac{\ln ^{2} y}{y^{3}}\right) .
\end{aligned}
$$

At the IR $\operatorname{limit}(\rho \rightarrow \infty)$, the metric (1.1) becomes

$$
\begin{gathered}
d s^{2}=R_{I R}^{2}\left(\frac{d v^{2}}{v^{2}}+\frac{v^{2}}{R_{I R}^{4}}\left(-d t^{2}+\sum_{i=1}^{3} d x_{i} d x_{i}\right)+d \Omega_{5}\right) \\
\phi=\phi_{\infty}, \quad R_{I R}^{2}=2^{-\frac{3}{4}} Q^{\frac{1}{2}} e^{\frac{1}{2} \phi_{\infty}}, \quad v=2^{-\frac{1}{2}} e^{\frac{1}{2} \phi_{\infty}} \rho^{-\frac{1}{4}}
\end{gathered}
$$

and this also describes $A d S_{5} \times S^{5}$ geometry.

In section 2 we study the D5-brane world volume action in the $N$ electric D3-brane background in the UV/IR regions and analyse the behavior of the tension at a spike. Section 3 is devoted to some comments about the baryon configuration.

\section{The D5-brane world volume action}

We consider the action of a D5-brane wrapped on $S^{5}$. The D5-brane world volume action consists of the Born-Infeld action and the WZ term. Since the Born-Infeld action in the Type 0 string theory is given in [5] [10], we can write down D5-brane world volume action

$$
\begin{aligned}
& S=-T_{5} \int d^{6} \sigma h\left(\frac{T}{2}\right) e^{-\phi} \sqrt{-\operatorname{det}\left(g_{a b}+F_{a b}\right)}+T_{5} \int A_{(1)} \wedge d C_{(4)}, \\
& h\left(\frac{T}{2}\right)=1+\frac{T}{4}+\frac{3 T^{2}}{32}+\cdots
\end{aligned}
$$


where $g_{a b}\left(=G_{\mu \nu} \partial_{a} X^{\mu} \partial_{b} X^{\nu}\right)$ is an induced metric, $F_{a b}\left(=d A_{(1)}\right)$ is a $\mathrm{U}(1)$ gauge field strength on the D5-brane and $C_{(4)}$ is a four-form field. The $\sigma=\left(t, \theta, \theta_{1}, \theta_{2}, \theta_{3}, \theta_{4}\right)$ is a world volume coordinate of the D5-brane embedded on $S^{5}$. For simplicity we consider an $\mathrm{SO}(5)$ isometry in the $S^{5}$ and a static solution as [3] . Let $\rho$ and $A_{t}$ depend only on $\theta$ and all other fields be zero. In this setup the action (2.1) becomes

$$
S=T_{5} \Omega_{4} \int d t d \theta \sin ^{4} \theta e^{\xi-2 \eta}\left\{-h\left(\frac{T}{2}\right) \sqrt{e^{\phi-5 \eta}{\rho^{\prime}}^{2}+e^{\phi-\eta}-F_{t \theta}{ }^{2}}+4 A_{t} e^{\phi}\right\},
$$

where' denotes the $\theta$ derivative and $\Omega_{4}\left(=\frac{8 \pi^{2}}{3}\right)$ is the volume of unit four sphere. The gauge field equation of motion is obtained as

$$
\begin{aligned}
\frac{\partial}{\partial \theta} K(\theta) & =-4 e^{\xi-2 \eta+\phi} \sin ^{4} \theta \\
K(\theta) & \equiv-h\left(\frac{T}{2}\right) \sin ^{4} \theta e^{\xi-2 \eta} \frac{\partial_{\theta} A_{t}}{\sqrt{e^{\phi-5 \eta} \rho^{\prime 2}+e^{\phi-\eta}-\left(\partial_{\theta} A_{t}\right)^{2}}} .
\end{aligned}
$$

Partially integrating (2.2), substituting (2.4) into it and changing sign, we evaluate the action (2.2) in the tree level

$$
E_{\rho}=T_{5} \Omega_{4} \int d \theta \sqrt{e^{\phi-5 \eta}{\rho^{\prime}}^{2}+e^{\phi-\eta}} \sqrt{K(\theta)^{2}+h^{2} \sin ^{8} \theta e^{2 \xi-4 \eta}} .
$$

This depends only on the $\rho$ field.

\subsection{The UV region}

At the UV limit $(\rho \rightarrow 0)$ Eq.(2.5) is expressed by using Eq.(1.4) as

$$
T_{5} \Omega_{4} \int d \theta \sqrt{{v^{\prime}}^{2}+v^{2}} \sqrt{K(\theta)^{2}+\frac{Q^{2}}{4} h\left(-\frac{1}{2}\right)^{2} \sin ^{8} \theta},
$$

and Eq.(2.3) is written as

$$
\partial_{\theta} K(\theta)=-4 R_{U V}^{4} \sin ^{4} \theta .
$$

Since the right hand side of (2.7) is a function of only $\theta$ not $\rho, K(\theta)$ is solvable and is determined as

$$
\begin{aligned}
& K(\theta)=R_{U V}{ }^{4} k(\theta), \\
& k(\theta) \equiv \frac{3}{2}(\nu \pi-\theta)+\frac{3}{2} \sin \theta \cos \theta+\sin ^{3} \theta \cos \theta,
\end{aligned}
$$

where $0<\nu<1$ is an integral constant. These results are similar to those in the Type IIB case [3], where $\nu=0$ corresponds to the baryon vertex and the spike sticking out at 
$\theta=\pi$ has the tension equal to that of the $N$ fundamental strings. So in this paper mainly we concentrate on the tension at $\nu=0$ and $\theta=\pi$. Then Eq.(2.6) implies that the tension at $\theta=\pi$ is $T_{5} \Omega_{4}|K(\pi)|=T_{5} \Omega_{4} R_{U V} \frac{3 \pi}{2}$.

Next we consider in the near UV region $(\rho \ll 1)$. From Eqs.(1.3) and $(2.3)$, the behavior of the $K(\theta)$ is given as

$$
\begin{aligned}
K(\theta) & =e^{\xi-2 \eta+\phi} k(\theta), \\
& \approx\left(1-\frac{1}{y}-\frac{1}{2 y^{2}}(39 \ln y+27)+\cdots\right) \frac{Q g_{s}}{2} k(\theta),
\end{aligned}
$$

where $g_{s}=e^{\phi}$ is the string coupling. Substituting (1.3) into (2.5), we can evaluate the $E_{\rho}$

$$
\begin{aligned}
E_{\rho}= & T_{5} \Omega_{4} \int d \theta \sqrt{e^{\frac{39 \ln y-5}{y}-\frac{5(39 \ln y-38)}{2 y^{2}}+\cdots} v^{\prime 2}+e^{\frac{39 \ln y-1}{y}-\frac{39 \ln y-38}{2 y^{2}}+\cdots} v^{2}} \\
& \times \sqrt{\left\{\left(1-\frac{1}{y}-\frac{1}{2 y^{2}}(39 \ln y+27)+\cdots\right) \frac{Q g_{s}}{2} k(\theta)\right\}^{2}+e^{2 \xi-4 \eta} h^{2} \sin ^{8} \theta} .
\end{aligned}
$$

These imply that the tension of the D5-brane at $\theta=\pi$ and $\nu=0$ is expressed as 1

$$
\begin{aligned}
& T_{5} \Omega_{4}\left(1+\frac{1}{2 y}(39 \ln y-7)+\frac{1}{8 y^{2}}\left(1521(\ln y)^{2}-1092 \ln y+317\right)+\cdots\right) \frac{Q g_{s}}{2}|k(\pi)| \\
= & p(\rho) T_{5} \Omega_{4} \frac{Q g_{s}}{2}|k(\pi)| \\
& p(\rho) \equiv 1-\frac{39 \ln (-\ln \rho)-7}{2 \ln \rho}+\frac{1521(\ln (-\ln \rho))^{2}-1092 \ln (-\ln \rho)+317}{8(\ln \rho)^{2}}+\cdots .
\end{aligned}
$$

Note that this tension includes a coupling constant $g_{s}$ and seems almost zero because the leading effective gravity solution (1.3) is asymptotically free in the UV region,

$$
g_{s}=e^{\phi}=2^{15} Q^{-1}(-\ln \rho)^{-2-\frac{39}{\ln \rho}+\cdots} .
$$

But it is not a problem. The D5-brane's tension [5],

$$
T_{5}=\frac{1}{\sqrt{2}(2 \pi)^{5}\left(l_{s}\right)^{6} g_{s}},
$$

where $l_{s}$ is the string length, has also an inverse coupling. So effects of the couplings are canceled and the tension does not become almost zero in the UV region. The behavior of (2.9) is shown in fig. 1 .

1 At the spike, $v^{\prime}$ will dominate $v$. 


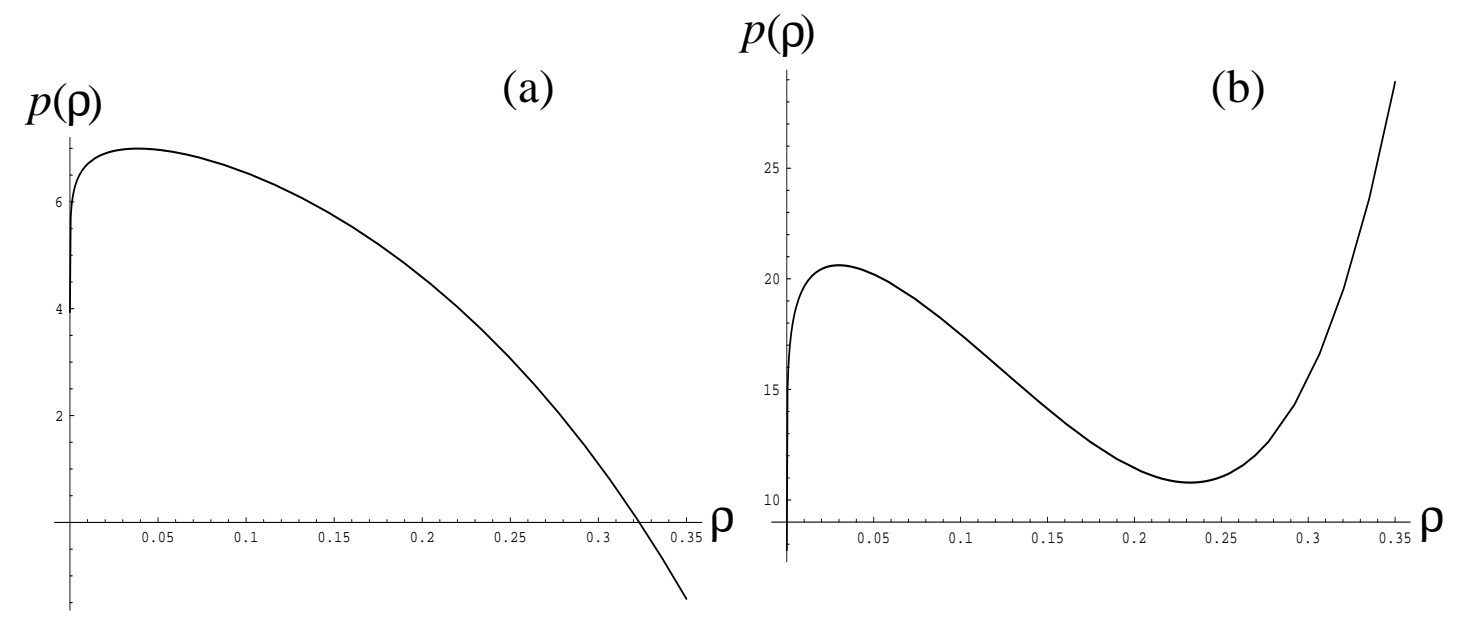

fig. 1 The behavior of $p(\rho)$ in the UV region $\rho \ll 1$.

In fig. 1, the (a) is the leading order approximation of $p(\rho)$, that is, $1-\frac{39 \ln (-\ln \rho)-7}{2 \ln \rho}$ and the (b) represents the second order approximation of $p(\rho)$, that is, $1-\frac{39 \ln (-\ln \rho)-7}{2 \ln \rho}+$ $\frac{1521(\ln (-\ln \rho))^{2}-1092 \ln (-\ln \rho)+317}{8(\ln \rho)^{2}}$. In the Type IIB case, the tension is constant since the radii of $S^{5}$ and $A d S_{5}$ are independent of $\rho$. On the other hand in the Type 0 case, the tension is not constant and flows as shown in fig. 1 since the radii of $S^{5}$ and $A d S_{5}$ depend on $\rho$ [7] . The (a) implies that there exist two points $\rho=0$, and $\rho=\exp \left(-\exp \left(\frac{7}{39}\right)\right) \approx$ 0.302219 , where $p(\rho)=1$. The latter point is an interesting and non trivial point, but it is less credible since in this section the $\rho \ll 1$ region is analyzed. In fact this point vanishes in the second order approximation in the (b). From these analyses, we conclude that the tension increases away from the UV limit. We will give more comments in section 3.

\subsection{The IR region}

Since at the IR limit the metric (1.6) describes $A d S_{5} \times S^{5}$ geometry as in the UV limit, the similar behavior will occur also in the IR region.

At the IR limit $(\rho \rightarrow \infty)$ the action (2.2) is calculated by Eq.(1.6)

$$
\int d \theta \sqrt{{v^{\prime}}^{2}+v^{2}} \sqrt{K(\theta)^{2}+\frac{Q^{2}}{8} h(0)^{2} \sin ^{8} \theta}
$$

and Eq.(2.3) is expressed as

$$
\partial_{\theta} K(\theta)=-4 R_{I R}^{4} \sin ^{4} \theta
$$


In the same way as the UV case, we concentrate on the tension at $\theta=\pi$ with $\nu=0$. Then Eq.(2.11) implies that the tension at the spike is $T_{5} \Omega_{4} R_{I R} \frac{3 \pi}{2}$. Since the $R_{I R}, R_{U V}$ have the following expressions,

$$
R_{U V}{ }^{4}=\frac{Q}{2} g_{s}, \quad R_{I R}{ }^{4}=\frac{Q}{2 \sqrt{2}} g_{s}
$$

the ratio of tensions at the UV and the IR limits is tured out to be $\frac{1}{\sqrt{2}}$.

Next we consider at the near IR region $(\rho \gg 1)$. Substituting (1.5) into (2.3), we obtain the asymptotic form of the $K(\theta)$

$$
K(\theta) \approx\left(1+\frac{7}{y}+\frac{1}{2 y^{2}}(63 \ln y+33)+\cdots\right) \frac{Q g_{s}}{2 \sqrt{2}} k(\theta)
$$

Substituting (1.5) into $(2.5)$, we calculate the $E_{\rho}$

$$
\begin{aligned}
E_{\rho}= & T_{5} \Omega_{4} \int d \theta \sqrt{e^{-\frac{9 \ln y+5}{y}-\frac{5(9 \ln y-2)}{2 y^{2}}+\cdots} v^{\prime 2}+e^{-\frac{9 \ln y+1}{y}-\frac{9 \ln y-2}{2 y^{2}}+\cdots} v^{2}} \\
& \times \sqrt{\left\{\left(1+\frac{7}{y}+\frac{1}{2 y^{2}}(63 \ln y+33)+\cdots\right) \frac{Q g_{s}}{2 \sqrt{2}} k(\theta)\right\}^{2}+e^{2 \xi-4 \eta} h^{2} \sin ^{8} \theta} .
\end{aligned}
$$

From these equations the tension at $\theta=\pi$ is obtained as

$$
\begin{aligned}
& T_{5} \Omega_{4}\left(1-\frac{1}{2 y}(9 \ln y-9)+\frac{1}{8 y^{2}}\left(81(\ln y)^{2}+37\right)+\cdots\right) \frac{Q g_{s}}{2 \sqrt{2}}|k(\pi)| \\
= & q(\rho) T_{5} \Omega_{4} \frac{Q g_{s}}{2 \sqrt{2}}|k(\pi)|, \\
& q(\rho) \equiv 1-\frac{9 \ln \ln \rho-9}{2 \ln \rho}+\frac{81(\ln \ln \rho)^{2}+37}{8(\ln \rho)^{2}}+\cdots .
\end{aligned}
$$

Note that this tension seems to diverge apparently since the string coupling $g_{s}$ behaves

$$
g_{s}=e^{\phi}=2^{-\frac{1}{2}} Q^{-1}(\ln \rho)^{2-\frac{9}{\ln \rho}+\cdots}
$$

But for the same reason as the UV case, the coupling $g_{s}$ is canceled with the one in the tension (2.10) . The behavior of (2.14) is shown in fig. 2 . 


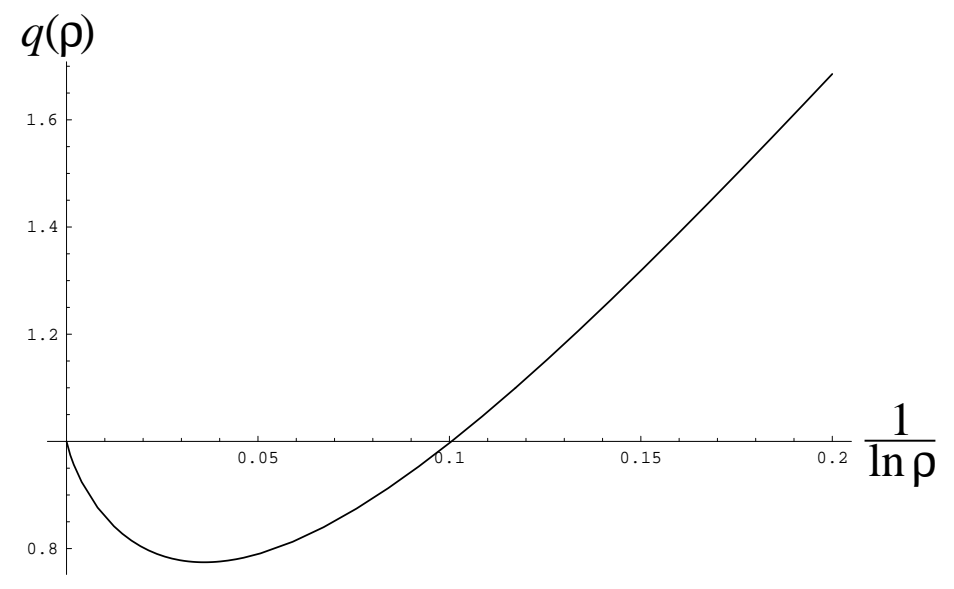

fig. 2 The behavior of $q(\rho)$ in the IR region $(\rho \gg 1)$.

In fig. 2 there are also two points $\rho=\infty$ and numerically $\rho=2.08581 \times 10^{4}$, where $q(\rho)=1$. This is the same situation as the UV case. Note that the second point is not reliable, because the IR region has a very large coupling and the perturbative analysis is no longer credible.

\section{Discussion}

In this paper we studied the static solution of D5-brane worldvolume action in the $\mathrm{UV} / \mathrm{IR}$ regions in the background with large $N$ electric D3-branes.

In the Type IIB case a relation $R^{4}=4 \pi g_{s} N l_{s}^{4}$ is satisfied, where $R$ is the radius of $A d S_{5}$ and $S^{5}$ and the $N$ is the number of D3-branes. This number comes from the action $\int d^{10} x \sqrt{G}\left(\cdots-\frac{1}{5 !} F_{5}^{2}\right)$ associated with the five form field strength. On the other hand, at the UV limit of the Type 0B, a relation $R_{U V}{ }^{4}=\frac{Q}{2} g_{s}$ is obtained from Eq.(1.4) up to normalization and the five form field strength appears in the action [5], in a form,

$$
\int d^{10} x \sqrt{G}\left(\cdots-f(T) \frac{1}{5 !} F_{5}^{2}\right) .
$$

This reads that $f(T)^{-\frac{1}{2}}$ contributes to $N$ in the Type 0 case (cf. (1.2) ). So using the same normalization as the type IIB case, we can calculate the $R_{U V}{ }^{4}$ as,

$$
R_{U V^{4}}=4 \pi g_{s} \frac{N}{f(-1)^{\frac{1}{2}}} l_{s}^{4}=4 \sqrt{2} \pi g_{s} N l_{s}^{4} .
$$


In the same way for the IR limit, we obtain

$$
R_{I R}{ }^{4}=4 \pi g_{s} \frac{N}{f(0)^{\frac{1}{2}}} l_{s}^{4}=4 \pi g_{s} N l_{s}^{4} .
$$

Eqs.(3.1) and (3.2) are consistent with the (2.13) . Then from Eqs.(2.10) and (3.1), the tension becomes

$$
T_{5} \Omega_{4} R_{U V}{ }^{4}|k(\pi)|=\frac{N}{2 \pi l_{s}^{2}}=N T_{f},
$$

where $T_{f}$ is a tension of a fundamental string. This corresponds to the bound state of $N$ quarks in $\mathrm{SU}(N)$ gauge theory, i.e. a baryon. As mentioned in section 2.1, in the UV region, there exist two points, the UV limit point $\rho=0$ and the non trivial one $(\rho=0.268067)$, where the tension is equal to $N T_{f}$. But the latter is less credible, because Eq.(2.9) is reliable only in small $\rho$ region and in fact the second point disappears in the analysis including the second order approximation. It would be interpreted as some perturbative effects.

Since in the IR limit, from Eqs. 2.10) and (3.2), we obtain the formula of tension,

$$
T_{5} \Omega_{4} R_{I R}^{4}|k(\pi)|=\frac{N}{2 \sqrt{2} \pi l_{s}^{2}}=\frac{N}{\sqrt{2}} T_{f} .
$$

In this case, the number of fundamental strings is not integer and this state is not a standard baryon. It may represent some quantum effects in such a confinement region with a strong coupling.

If the flow of tension is connected smoothly from the UV limit to the IR limit, there must exist at least one point where a baryon configuration with $N$ quarks is realised, since the flow increases near the UV limit. But we are not able to determine this interesting point exactly, since the full metric of D3-brane background has not been known yet and we cannot discuss how the UV solution is connected with the IR solution. If the full metric is found, the behavior of that non trivial point will be clarified.

Though in this paper the background with only the $N$ electric D3-branes is discussed, this method will be applicable to more general backgrounds, for example, a model with $N$ electric and $N$ magnetic D3-branes, in which case the dual theory is an $\mathrm{SU}(N) \times \mathrm{SU}(N)$ Yang-Mills theory [11] [12].

\section{Acknowledgement}

I am grateful to K.Sugiyama for useful discussions. This work is supported in part by JSPS Research Fellowships for Young Scientists (\#4783). 


\section{References}

[1] E.Witten, "Baryons and Branes in Anti de Sitter Space," JHEP 9807 (1998) 006, hep-th/9805112.

[2] D.Gross and H.Ooguri, "Aspects of Large $N$ Gauge Theory Dynamics as seen by String Theory," Phys.Rev. D58 (1998) 106002, hep-th/9805129.

[3] C.G.Callan, A.Güijosa and K.G.Savvidy, "Baryons and String Creation from the Fivebrane Worldvolume Action," hep-th/9810092; C.G.Callan, A.Guijosa, K.G.Savvidy and O.Tafjord, "Baryons and Flux Tubes in Confining Gauge Theories from Brane Actions," hep-th/9902197.

[4] C.G.Callan and J.M.Maldacena, "Brane Dynamics From the Born-Infeld Action," Nucl.Phys. B513 (1998) 198-212, hep-th/9708147.

[5] I.R.Klebanov and A.A.Tseytlin, "D-Branes and Dual Gauge Theories in Type 0 Strings," Nucl.Phys. B546 (1999) 155-181, hep-th/9811035.

[6] J.Polchinski, "String Theory," vol. 2, Cambridge University Press, 1998.

[7] I.R.Klebanov and A.A.Tseytlin, "Asymptotic Freedom and Infrared Behavior in the Type 0 String Approach to Gauge Theory," Nucl.Phys. B547 (1999) 143-156, hepth/9812089.

[8] J.A.Minahan, "Glueball Mass Spectra and Other Issues for Supergravity Duals of QCD Models," JHEP 9901 (1999) 020, hep-th/9811156

[9] J.A.Minahan, "Asymptotic Freedom and Confinement from Type0 String Theory," JHEP 9904 (1999) 007, hep-th/9902074.

[10] M.R.Garousi, "String Scattering form D-branes in Type 0 Theories," hep-th/9901085.

[11] I.R.Klebanov and A.A.Tseytlin, "A Non-supersymmetric Large N CFT from Type 0 String Theory," JHEP 9903 (1999) 015, hep-th/9901101.

[12] A.A.Tseytlin and K.Zarembo, "Effective Potential in Non-supersymmetric $S U(N) \times$ $S U(N)$ Gauge Theory and Interactions of Type 0 D3-branes," hep-th/9902095. 